\title{
Ferramentas Assistivas no Ensino e Aprendizagem de Crianças com Aspectro Autista: Um Mapeamento Sistemático
}

\author{
Rodrigo Feitosa Gonçalves ${ }^{1}$, Clinton Hudson Moreira Pessoa ${ }^{1}$, \\ Odette Mestrinho Passos ${ }^{1}$, Rainer Xavier de Amorim ${ }^{1}$ \\ ${ }^{1}$ Instituto de Ciências Exatas e Tecnologia - Universidade Federal do Amazonas \\ (UFAM) \\ \{feitosar9, clintonhudson97\}@gmail.com; \{odette,raineramorim\}@ufam.edu.br

\begin{abstract}
Several tools have been designed to meet educational demands caused by Autism Spectrum Disorder (ASD). Therefore, this article aims to present the main assistive tools that provide support to the teaching and learning of children with ASD, adopting for this, a research methodology based on the principles of Experimental Software Engineering, which is based on the conduction of a study secondary: Systematic Mapping. As a result, 17 assistive tools were identified, the majority of which were contextualized to aid literacy and 5 pedagogical approaches applied in 59\% of the mapped tools.
\end{abstract}

Resumo. Diversas ferramentas têm sido projetadas para atender demandas educativas causadas pelo Transtorno do Espectro Autista (TEA). Diante disso, este artigo tem como objetivo apresentar as principais ferramentas assistivas que fornecem apoio ao ensino e aprendizagem de crianças com TEA, adotando para isso, uma metodologia de pesquisa fundamentada nos princípios da Engenharia de Software Experimental, que se baseia na condução de um estudo secundário: o Mapeamento Sistemático. Como resultado foram identificas 17 ferramentas assistivas, sendo a maioria contextualizada para o auxílio da alfabetização e 5 abordagens pedagógicas, aplicadas em $59 \%$ das ferramentas mapeadas.

\section{Introdução}

O vasto surgimento de softwares voltados para educação tem possibilitado inúmeras oportunidades de incrementar e aperfeiçoar o aprendizado de crianças por meio de ferramentas que apresentam conteúdos cada vez mais dinâmicos, com animações e representações gráficas e que representam uma possibilidade de inovação nos recursos didáticos [Lima, 2013]. Não obstante, no processo de inclusão social e aprendizagem de crianças com Transtorno do Espectro Autista (TEA) muitos estudos têm sido desenvolvidos na tentativa de oportunizar softwares assistivos que sejam capazes de atender as necessidades, auxiliar e atuar como um meio de apoio [Moura et al., 2016; Lima et al., 2013].

As características do TEA se apresentam na persistência no déficit de comunicação e interação social e pelos padrões de comportamento, de interesse e de atividades que são restritos e repetitivos. O termo "Espectro" corresponde à multiplicidade de sintomas e comprometimentos possíveis e engloba o Transtorno Autista, a Síndrome de Asperger e o Transtorno Invasivo do Desenvolvimento NãoEspecificado [APA, 2013]. 
VIII Congresso Brasileiro de Informática na Educação (CBIE 2019)

Anais do XXX Simpósio Brasileiro de Informática na Educação (SBIE 2019)

O TEA se caracteriza pelos prejuízos persistentes na comunicação e interação social, bem como nos comportamentos que podem incluir os interesses e os padrões de atividades, sintomas que estão presentes desde a infância e limitam ou prejudicam o funcionamento diário do indivíduo [APA, 2013]. Dessa forma, atividades educacionais do cotidiano educacional devem ser adaptadas para cada caso, possibilitando o desenvolvimento do indivíduo e aumentando suas capacidades [Ferreira et al., 2018].

Diante do contexto, os aplicativos, através de smartphones, por exemplo, tem se configurado como instrumento relevante para diminuir a segregação e facilitar o ensino de aprendizado de indivíduos, além de ser uma alternativa interessante para auxiliar no ensino e aprendizagem de pessoas com TEA [Moura et al., 2016].

A metodologia de pesquisa adotada neste trabalho, para coletar as informações de forma a cumprir o objetivo, está fundamentado nos princípios da Engenharia de Software Experimental que se baseia na condução de um estudo secundário: Mapeamento Sistemático (MS). O MS fornece uma visão geral de uma área de pesquisa, identificando a quantidade, os tipos de pesquisas realizadas, os resultados disponíveis, além das frequências de publicações ao longo do tempo para identificar tendências [Petersen et al., 2008].

Kitchenham e Charters (2007), afirmam que estudos de MS têm sido recomendados, sobretudo para áreas de pesquisa onde é difícil visualizar a gama de materiais, relevantes e de alta qualidade, que possam estar disponíveis. Assim sendo, a escolha do MS como proposta para a condução desta pesquisa, justifica-se pelo fato do objetivo da pesquisa ser apenas identificar e utilizar os resultados obtidos para futuras pesquisas.

Dessa forma, este artigo tem como objetivo apresentar as principais ferramentas assistivas que auxiliam no ensino e aprendizagem de crianças com TEA, com a finalidade de catalogar e identificar as características dessas ferramentas, assim como as abordagens pedagógicas adotadas pelas mesmas.

O restante do artigo está organizado da seguinte maneira: a Seção 2 apresenta a os conceitos relacionados e discute os trabalhos relacionados. A Seção 3 apresenta a metodologia adotada (Mapeamento Sistemático), enquanto a Seção 4 mostra os resultados e as discussões. A Seção 5 apresenta as considerações finais e as propostas de trabalhos futuros.

\section{Referencial Teórico}

\subsection{Conceitos Relacionados}

O TEA é descrito como uma perda nas interações sociais por parte do indivíduo, sendo caracterizada por um dos seguintes itens: a) dificuldade com o uso de comportamento não verbal; b) pouco contato visual com outros indivíduos; c) bloqueio em desenvolver relações com colegas; d) Desestímulo em compartilhar interesses e prazeres com terceiros e e) Ausência de reciprocidade emocional e social [Ishihara, Tamanaha e Perissinoto, 2016].

Pesquisas têm evidenciado que softwares e tecnologia tendem a ser bem recebidos no apoio ao ensino e aprendizado de pessoas com TEA [Putnam, 2008]. Além disso, nos últimos anos, se comprovam a efetividade de treinamentos baseados em 
VIII Congresso Brasileiro de Informática na Educação (CBIE 2019)

Anais do XXX Simpósio Brasileiro de Informática na Educação (SBIE 2019)

computadores para desenvolvimento cognitivo e melhoria das habilidades de crianças autistas na educação [Sousa, Costa e Castro, 2012].

Os motivos do aceitamento demonstrados pelas pesquisas incluem: a) Programas de computador que dispõem de uniformidade para os indivíduos previsíveis e familiares, b) Atividades repetitivas dispostas de forma fácil e sem grandes mudanças, c) Softwares e treinamentos sem interações sociais complexas com terceiros, o que possibilita ao usuário com TEA trabalhar na sua própria velocidade [Powell, 1996] e d) Ferramentas educacionais para computadores pessoais que proporcionam um ambiente de aprendizado estruturado e individual, que na maioria das vezes é necessário para uma criança TEA aprender um novo tópico com efetividade [Ferreira et al., 2018].

A forma com que as características e sintomas desse transtorno se apresentam nas pessoas com TEA, deixa claro que há uma grande importância em incentivar crianças ao uso de softwares que visem proporcionar maior autonomia e independência das mesmas. Vale ressaltar, que as atividades devem ser adaptadas a realidade de cada indivíduo, respeitando suas individualidades e grau de comprometimento provocado pelo transtorno [Lima, 2013].

\subsection{Trabalhos Relacionados}

O trabalho de Ferreira et al. (2018) teve como objetivo identificar como as pesquisas desenvolvidas e disseminadas em eventos acadêmicos nacionais têm contemplado a tríade "Autismo, Educação e Tecnologia". Para isso, foi adotada uma revisão de literatura por meio de publicações nacionais em anais de conferências e em revistas que abordam temas relacionados à Inclusão, Acessibilidade, Educação e Tecnologia.

Após análise das informações obtidas, os pesquisadores puderam identificar que as pesquisas executadas estão em consonância com as diretrizes do governo, ou seja, há um expressivo grupo de soluções de software destinadas à Educação (principalmente para letramento e alfabetização) e que abordam os modelos práticos de intervenção consolidados nas abordagens clássicas [Ferreira et al., 2018].

O trabalho de Silva, Moura e Soares (2017) teve como objetivo identificar e investigar as tendências e lacunas dos avanços no uso de tecnologias computacionais para o ensino de crianças com TEA. Para alcançar o objetivo principal, foi realizada um Mapeamento Sistemático da Literatura, por meio de publicações de bibliotecas digitais.

A partir das análises dos resultados das questões secundárias, os pesquisadores identificaram que as ferramentas tecnológicas expostas nos trabalhos levantados possibilitam o desenvolvimento no ensino de crianças com autismo, isso porque a maioria das ferramentas identificadas se caracterizou como mobile, gerando facilidade de acesso das ferramentas. Constou-se ainda que há uma grande ausência de ferramentas que utilizam Inteligência Artificial [Silva, Moura e Soares, 2017].

As pesquisas de Ferreira et al. (2018) e Silva, Moura e Soares (2017) se assemelham a este trabalho por apresentarem ferramentas e levantamento de trabalhos voltadas para o auxílio de crianças com TEA. Entretanto, na pesquisa de Ferreira et al. (2018), houve apenas o foco no levantamento de trabalhos publicados em âmbito nacional, não levando em consideração a avaliação das características dos softwares apresentados. Já o trabalho de Silva, Moura e Soares (2017) ficou restrito a uma faixa temporal de buscas de apenas 6 (seis) anos, datados do ano de 2011 a 2016. Outro ponto 
VIII Congresso Brasileiro de Informática na Educação (CBIE 2019)

Anais do XXX Simpósio Brasileiro de Informática na Educação (SBIE 2019)

deste trabalho que vale ressaltar é o fato do mesmo apresentar apenas buscas em bibliotecas digitas.

O presente trabalho, diferentemente dos acima citados, não possui uma faixa limite de tempo para as buscas realizadas nos simpósios e revistas utilizados para a pesquisa, apresentando buscas nos trabalhos em todos os anos em que os mesmos ocorreram. O mesmo apresenta ainda as características pedagógicas e as diretrizes adotadas no desenvolvimento das ferramentas mapeadas.

\section{Mapeamento Sistemático}

O MS foi baseado no guidelines desenvolvido por Kitchenham e Chartes (2007) e definido em três etapas: (a) Planejamento do Mapeamento: nesse passo, os objetivos da pesquisa são listados e o protocolo do mapeamento é definido; (b) Condução do Mapeamento: durante essa fase, as fontes para o mapeamento são selecionadas, os estudos são identificados, selecionados e avaliados de acordo com os critérios estabelecidos no protocolo do mapeamento e (c) Resultado do Mapeamento: nessa fase, os dados dos estudos são extraídos e sintetizados para serem publicados.

O objetivo deste mapeamento sistemático foi analisar publicações científicas com o propósito de identificar as principais ferramentas assistivas que podem auxiliar no processo de ensino e aprendizagem de crianças com TEA. Sendo assim, este MS buscou respostas para as seguintes questões de pesquisa: [QP1]: Quais as ferramentas assistivas para apoiar o ensino e a aprendizagem de crianças com TEA? e [QP2]: Quais abordagens pedagógicas adotadas para o desenvolvimento das ferramentas assistivas?

Os locais de buscas definidos para a pesquisa foram feitos a partir da busca manual nos anais do Simpósio Brasileiro de Informática na Educação (SBIE), Workshop de Informática na Escola (WIE), Concurso Integrado de Desenvolvimento de Soluções de Tecnologia e Objetos de Aprendizagem para a Educação (Apps.Edu), Workshop de Psicopedagogia e Tecnologias Digitais (WPTD), Simpósio Brasileiro de Jogos e Entretenimento Digital (SBGames), Revista Novas Tecnologias na Educação (RENOTE) e Revista Brasileira de Informática na Educação (RBIE).

A busca foi restringida usando-se palavras-chave específicas para encontrar as publicações de interesse sendo composta a partir da combinação de seus sinônimos usando os operadores lógicos OR e AND: ((educação OR ensino OR aprendizagem OR alfabetização OR letramento) OR (ferramenta OR sistema OR aplicação OR software OR ambiente OR jogo)) AND (Autismo OR distúrbio do aspectro autista OR déficit de atenção OR Aspectro OR distúrbio hiperativo OR autista OR TEA)

A pesquisa se restringiu à análise de publicações disponíveis até a data presente da execução do estudo. A seleção das publicações foi realizada em quatro etapas: (1) Busca preliminar das publicações coletadas nas fontes definidas, (2) Análise do título, do resumo e das palavras-chave, (3) Leitura completa e (4) Extração dos dados.

Para que a etapa (3) fosse realizada, foi adotado um critério de inclusão, onde a publicação deveria abordar o desenvolvimento de uma ferramenta assistiva para o apoiar o ensino e a aprendizagem de crianças com TEA.

Foram excluídas publicações não relevantes para as questões investigadas e na dúvida quanto à inclusão ou exclusão de algum estudo, o mesmo foi mantido para leitura na íntegra. Tendo como objetivo observar a evolução das pesquisas 
VIII Congresso Brasileiro de Informática na Educação (CBIE 2019)

Anais do XXX Simpósio Brasileiro de Informática na Educação (SBIE 2019)

desenvolvidas na área no decorrer dos anos, este MS não restringiu sua busca a um intervalo específico, entretanto somente publicação em inglês e português foram aceitas.

Na etapa da Condução do MS, a execução ocorreu entre os meses de abril a junho de 2019, e as publicações foram selecionadas de acordo com o critério estabelecido no protocolo. Publicações duplicadas, inacessíveis ou indisponíveis na internet foram descartadas.

De um total de 114 publicações identificadas, após a leitura do título, resumo e palavras-chave, 41 foram selecionadas seguindo o perfil da pesquisa. Todas elas foram lidas na íntegra e ao final 17 publicações foram selecionadas por atenderem o critério de inclusão, apresentados na Tabela 1, assim como no protocolo disponível em: http://www.icet.ufam.edu.br/grupopesq/gptec/lab312/pub/reltec/protocolo_mstea.pdf.

Tabela 1: Estudos Primários por base de busca

\begin{tabular}{|c|c|c|c|c|}
\hline Fontes & Ano & Inicialmente & $\begin{array}{c}\text { Resultado } \\
\text { da Etapa 1 }\end{array}$ & $\begin{array}{c}\text { Resultado } \\
\text { da Etapa 2 }\end{array}$ \\
\hline SBIE & $2001-2018$ & 33 & 15 & 3 \\
\hline WIE & $2003-2018$ & 23 & 4 & 1 \\
\hline APPs.Edu & $2015-2018$ & 19 & 5 & 5 \\
\hline WPTD & 2016 & 1 & 3 & 1 \\
\hline SBGAMES & $20014-2018$ & 23 & 10 & 6 \\
\hline RENOTE & $2003-2018$ & 5 & 1 & 1 \\
\hline RBIE & $1997-2018$ & 10 & 3 & 0 \\
\hline \multicolumn{2}{r|}{ TOTAL } & $\mathbf{1 1 4}$ & $\mathbf{4 1}$ & $\mathbf{1 7}$ \\
\hline
\end{tabular}

\section{Resultados e Discussões}

Com relação à primeira questão de pesquisa "Quais as ferramentas assistivas para apoiar o ensino e a aprendizados de crianças com TEA?" foram identificadas 17 ferramentas conforme pode ser observado na Tabela 2.

Ao analisar a Tabela 2 é possível observar que das 17 ferramentas, 10 (59\%) são destinados ao apoio inicial da alfabetização de crianças com TEA. A publicação [FA8], além de apresentar apoio no ensino de português, também aborda o ensino de matemática através de ideias sobre unidades, dezenas, soma e subtração.

Em relação a característica "Adaptabilidade" que se refere ao fato da ferramenta ser adaptada para cada grau de autismo, é possível verificar que 5 publicações se apresentam como adaptativas ([FA1], [FA4], [FA5], [FA12] e [FA13]). Essa característica possui uma grande vantagem, pois segundo [Ferreira et al., 2018] as atividades do cotidiano educacional de indivíduos com TEA devem ser adaptadas para cada caso, possibilitando o desenvolvimento do indivíduo e aumentando suas capacidades de aprendizagem.

Constatou-se ainda que três $(18 \%)$ das ferramentas identificadas se caracterizam como aspecto comunicacional, o que simboliza um aspecto positivo, pois é necessário que ocorra o incentivo da comunicação entre indivíduos com TEA através de atividades educacionais do cotidiano desde a infância [Ferreira et al., 2018]. Outro ponto importante identificado neste MS foi que $59 \%$ das ferramentas assistivas não especificou a faixa etária do público-alvo, e dentre os que informaram, a idade seria de 3 a 13 anos. 
VIII Congresso Brasileiro de Informática na Educação (CBIE 2019)

Anais do XXX Simpósio Brasileiro de Informática na Educação (SBIE 2019)

Tabela 2. Características das ferramentas assistivas

\begin{tabular}{|c|c|c|c|c|c|c|}
\hline ID & Título & Objetivo & Faixa Etária & $\begin{array}{r}\text { Adapta } \\
\text { bilidade }\end{array}$ & Ano & Evento \\
\hline FA1 & Educautism & $\begin{array}{c}\text { Auxilia na } \\
\text { alfabetização }\end{array}$ & NE & Sim & 2018 & RENOTE \\
\hline FA2 & - & Comunicação & De 5 a 9 anos & $\mathrm{NE}$ & 2011 & SBGAMES \\
\hline FA3 & G-TEA & Ensino de cores & $\mathrm{NE}$ & $\mathrm{NE}$ & 2013 & SBGAMES \\
\hline FA4 & ComFiM & Comunicação & Diferentes idades & Sim & 2014 & SBGAMES \\
\hline FA5 & ABC Fônico & $\begin{array}{c}\text { Auxilia na } \\
\text { alfabetização }\end{array}$ & De 4 a 8 anos & Sim & 2018 & SBGAMES \\
\hline FA6 & Projeto Autasy & Histórias infantis & $\mathrm{NE}$ & $\mathrm{NE}$ & 2018 & SBGAMES \\
\hline FA7 & ACA & $\begin{array}{c}\text { Auxilia a } \\
\text { alfabetização }\end{array}$ & De 5 a 7 anos & $\mathrm{NE}$ & 2018 & SBGAMES \\
\hline FA8 & TEO & $\begin{array}{c}\text { Ensino } \\
\text { multidisciplinar }\end{array}$ & $\mathrm{NE}$ & NE & 2016 & SBIE \\
\hline FA9 & LIA & $\begin{array}{l}\text { Narrativa de } \\
\text { histórias }\end{array}$ & De 7 a 13 anos & NE & 2017 & SBIE \\
\hline FA10 & Alfa Autista & $\begin{array}{c}\text { Auxilia na } \\
\text { alfabetização }\end{array}$ & NE & NE & 2018 & SBIE \\
\hline FA11 & ABC Autismo & $\begin{array}{c}\text { Auxilia na } \\
\text { alfabetização }\end{array}$ & NE & NE & 2015 & APPS.Br \\
\hline FA12 & SCALA Tablet & $\begin{array}{c}\text { Comunicação e } \\
\text { letramento }\end{array}$ & De 3 e 5 anos & Sim & 2015 & APPS.Br \\
\hline FA13 & HangAut Game & $\begin{array}{c}\text { Auxilia na } \\
\text { alfabetização }\end{array}$ & $\mathrm{NE}$ & Sim & 2016 & APPS.Br \\
\hline FA14 & Litera Azul & $\begin{array}{c}\text { Auxilia na } \\
\text { alfabetização }\end{array}$ & De 6 a 10 anos & NE & 2016 & APPS.Br \\
\hline FA15 & MusicApp & Música & $\mathrm{NE}$ & $\mathrm{NE}$ & 2016 & APPS.Br \\
\hline FA16 & RA móvel & $\begin{array}{c}\text { Auxilia na } \\
\text { alfabetização }\end{array}$ & $\mathrm{NE}$ & $\mathrm{NE}$ & 2016 & WPTD \\
\hline FA17 & SwAspie & $\begin{array}{c}\text { Auxilia na } \\
\text { alfabetização }\end{array}$ & $\mathrm{NE}$ & $\mathrm{NE}$ & 2017 & WIE \\
\hline
\end{tabular}

Legenda: NE - Não especificado

Tendo como objetivo investigar o progresso de pesquisas relacionadas ao desenvolvimento de tecnologias assistivas para apoiar o ensino e aprendizagem de indivíduos com TEA, foi elaborado um gráfico que apresenta a distribuição das publicações analisadas neste MS agrupadas por ano de publicação (Figura 1). A partir do resultado obtido, constatou-se que apesar da busca não ter sido limitada por um período de tempo, o primeiro estudo publicado nas fontes adotadas, foi em 2011, o que indica que estudos nesta área são relativamente novos.

Entre os anos de 1997 a 2010 não houve nenhuma publicação acerca de tema da pesquisa. Após 3 anos tendo somente um estudo, nos anos de 2016 e 2018, ocorreu um crescimento no número de publicações relacionadas as ferramentas assistivas para crianças com TEA, constatando uma ligeira tendência da comunidade científica em desenvolver pesquisas na área. 
VIII Congresso Brasileiro de Informática na Educação (CBIE 2019)

Anais do XXX Simpósio Brasileiro de Informática na Educação (SBIE 2019)

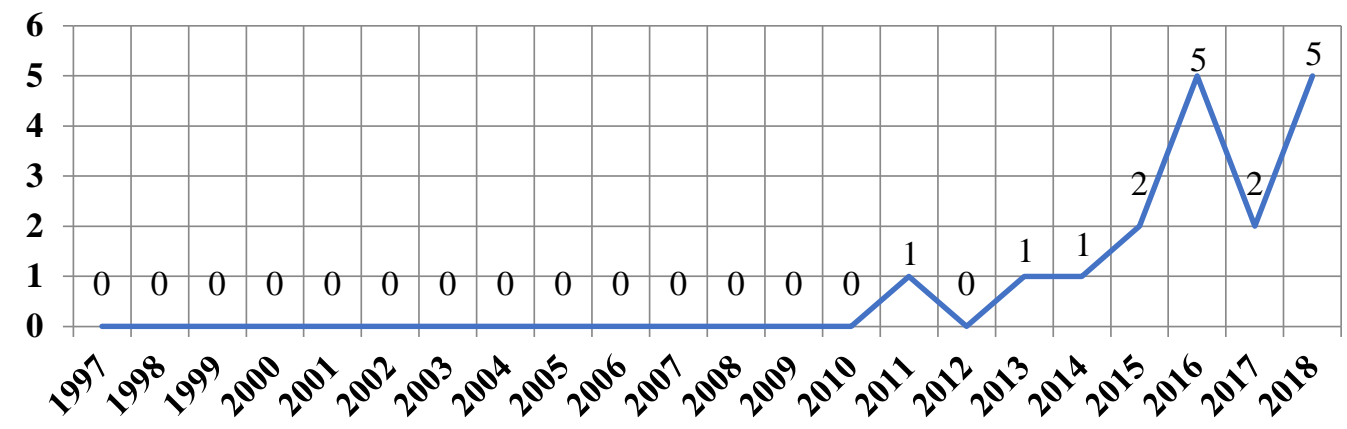

Figura 1. Quantitativo de publicações por ano

A Tabela 3 apresenta as categorias de divisão de cada ferramenta, assim como a Tabela 4 mostra os dispositivos para qual as ferramentas foram desenvolvidas. Em relação as categorias, constatou-se que houve a predominância de jogos, seguido por ambientes digitais de aprendizagem, que disponibilizam conteúdos instrucionais ilustrativos e atividades didático-pedagógicas como recurso para motivar o aluno através de animações.

Pode-se observar, ainda, que a $88 \%$ das ferramentas são aplicativos, ou seja, estão disponíveis para dispositivos móveis, enquanto 4 são para plataforma desktop. Isso talvez se deva ao fato de que os aplicativos geram facilidades no acesso [Silva, Moura e Soares, 2017]. Dos 13 aplicativos, 6 são jogos, 4 são ambientes de aprendizagem e 3 são ferramentas multimídias.

Tabela 3. Categorias Identificadas

\begin{tabular}{|c|c|}
\hline Categorias & Publicações \\
\hline Ambiente de Aprendizagem & [FA9], [FA11], [FA12], [FA15] e [FA16] \\
\hline Ferramenta Multimídia & [FA3], [FA14] e [FA17] \\
\hline Jogo & [FA1], [FA2], [FA4], [FA5], [FA6], [FA7], [FA8], \\
[FA10] e [FA13]
\end{tabular}

Tabela 4. Dispositivos

\begin{tabular}{|c|c|}
\hline Dispositivos & Publicações \\
\hline Celular e Desktop & [FA1] e [FA11] \\
\hline Celular & [FA3], [FA4], [FA5], [FA7], [FA8], [FA9], [FA10], [FA12], [FA13], \\
\hline [FA14], [FA15], [FA16] e [FA17] \\
\hline Desktop & [FA2] e [FA6] \\
\hline
\end{tabular}

Quando se trabalha com o desenvolvimento de ferramentas educacionais é necessário definir uma abordagem para que dê suporte ao aspecto educativo dos mesmos. Quando se trata da educação de crianças com TEA, este aspecto é ainda mais importante considerando que, há necessidade de se considerar a condição de cada indivíduo com esse transtorno, para que seja possível a inclusão desse público na educação básica [Abreu et al., 2012].

Dessa forma com relação à segunda questão de pesquisa "Quais abordagens pedagógicas adotadas para o desenvolvimento das ferramentas assistivas?” foram identificadas 5 abordagens conforme pode ser observado na Tabela 5. Entretanto, é importante salientar, que somente dez estudos (59\%) adotaram uma abordagem. Esse dado destaca uma carência que pode limitar o potencial educacional das ferramentas desenvolvidas e afetar os benefícios destinados às crianças com TEA. 
VIII Congresso Brasileiro de Informática na Educação (CBIE 2019)

Anais do XXX Simpósio Brasileiro de Informática na Educação (SBIE 2019)

Há uma predominância da abordagem TEACCH como método pedagógico para o desenvolvimento das atividades nas ferramentas. Talvez isso seja pelo fato de que o TEACCH possibilita trabalhar na estruturação do tempo, atividades, materiais e ambientes utilizados pela criança objetivando compensar os déficits característicos do autismo e oportunizar ganhos significativos para o convívio social [Farias et al., 2015].

Tabela 5. Abordagens pedagógicas

\begin{tabular}{|l|c|}
\hline \multicolumn{1}{|c|}{ Abordagem Pedagógica } & Publicações \\
\hline ABA (Análise do Comportamento Aplicada) & [FA3] e [FA17] \\
\hline AVDs (Atividades de Vida Diária) & [FA8] \\
\hline FÔNICO (Ensina através de sons de cada letra) & [FA5] e [FA10] \\
\hline $\begin{array}{l}\text { TEACCH (Tratamento e Educação para Autistas e Crianças com } \\
\text { Déficits Relacionados com a Comunicação) }\end{array}$ & [FA7], [FA11], [FA13], \\
\hline $\begin{array}{l}\text { PECS (Comunicação baseada na comunicação Picture Exchange } \\
\text { System) }\end{array}$ & [FA4] \\
\hline
\end{tabular}

Quando se trabalha com desenvolvimento de software, os métodos e os procedimentos devem ser adotados para aumentar a produtividade e qualidade dos produtos. Nesse contexto, os produtos de software na temática educacional, além de envolver uma equipe multidisciplinar, devem absorver os objetivos educacionais propostos e o ambiente de aprendizagem desejado, adotando diretrizes e criando situações que estimulem o desenvolvimento de ferramentas que atenda às necessidades dos usuários [Araújo e Almeida, 2009]. Assim, de forma à complementar os resultados, a Tabela 6 mostra as diretrizes que foram adotadas no desenvolvimento das ferramentas assistidas identificadas.

Apesar das ferramentas identificadas apresentarem a mesma finalidade, ou seja, o apoio ao ensino-aprendizagem de crianças com TEA, as diretrizes adotadas em seu desenvolvimento foram diferentes, entretanto, podemos ressaltar que foi dada uma atenção aos aspectos computacionais para $53 \%$ da ferramentas.

Tabela 6. Diretrizes identificadas no desenvolvimento

\begin{tabular}{|l|c|}
\hline \multicolumn{1}{|c|}{ Diretrizes para Desenvolvimento } & Publicações \\
\hline Interação Humano-Computador (IHC) & {$[$ FA1] } \\
\hline Mecânica-Dinâmica-Estética (MDA) & {$[$ FA4] } \\
\hline Unified Game Canvas (UGC) & {$[\mathrm{FA6]}$} \\
\hline Técnicas computacionais para a análise e processamento de imagens & [FA7] e [FA14] \\
\hline Sistema de Comunicação por Figuras (PECS) & {$[\mathrm{FA} 8]$} \\
\hline Interação Humano-Computador (IHC) e Design de games & {$[\mathrm{FA9]}$} \\
\hline Padrões de projeto (Design Pattern) & {$[\mathrm{FA12}]$} \\
\hline Técnicas de realidade aumentada & {$[$ FA16] } \\
\hline
\end{tabular}

Outra questão relevante identificada foi quanto aos tipos de tecnologias de interface (realidade virtual, realidade aumentada e 3D/2D) utilizadas, que com base na análise das ferramentas, foi visto que apenas as publicações [FA3] e [FA16] especificaram o uso de 2D/3D como meio de interação e animação, se caracterizando como uma solução interessante para ferramentas que utilizam humanos virtuais como recurso computacional. Também foi notado que apenas uma ferramenta [FA16], adotou realidade aumentada e como classificador de imagens apenas os estudos [FA7] e [FA14], apresentando-se como uma oportunidade de ampliar a capacidade de representação visual das ferramentas.

A interação do usuário com o computador ocorre por meio de uma interface, onde verificou-se que $88 \%$ das ferramentas utilizaram a tecnologia Interface Gráficas do Usuário (GUI, em inglês, Graphical User Interface), como mostra a Tabela 7, que 
VIII Congresso Brasileiro de Informática na Educação (CBIE 2019)

Anais do XXX Simpósio Brasileiro de Informática na Educação (SBIE 2019)

emprega o método de imagens gráficas e widgets em contexto com o texto para representar as informações e ações disponíveis ao usuário. A GUI é tida como uma interface amigável por proporcionar um conjunto de componentes que possibilitam a utilização da aplicação.

Tabela 7. Tecnologia de interface

\begin{tabular}{|c|c|}
\hline Tecnologia de Interface & Publicações \\
\hline Animação 2D & [FA3] \\
\hline Classificador de imagens e GUI & [FA7] e [FA14] \\
\hline GUI & [FA1], [FA2], [FA4], [FA5], [FA6], [FA8], [FA9], [FA10], \\
\hline Realidade aumentada e 3D & [FA11], [FA12], [FA13], [FA15] e [FA17] \\
\hline
\end{tabular}

Atualmente há uma grande importância de se validar um produto de software não somente no seu desenvolvimento, mas também com seu usuário, assim obtendo uma ferramenta final capaz de auxiliar o educador e aluno no processo de ensino e aprendizagem. Isso caracteriza a avaliação de software educacional uma etapa indispensável na inserção destas ferramentas em sala de aula [Webber et al., 2009].

Com relação ao fato das ferramentas assistivas terem sido ou não validades/testadas, podemos afirmar que das 17, apenas 47\% ([FA1], [FA2], [FA3], [FA4], [FA8], [FA9], [FA10] e [FA13]), foram validadas/testadas especificamente com crianças que foram diagnosticadas com TEA, e 18\% ([FA5] e [FA12]) validadas/testadas com pais de crianças com TEA ou profissionais da área. As demais publicações não especificaram se houve ou não a validação com o usuário.

\section{Considerações Finais}

As tecnologias assistivas são utilizadas como instrumento de acessibilidade e inclusão, o qual visa integrar tecnologia e inclusão em uma ferramenta capaz de atender e auxiliar alunos com necessidades educacionais especiais. Assim, esse trabalho apresenta um mapeamento sistemático sobre ferramentas assistivas para apoiar o ensino e a aprendizagem de crianças com Transtorno do Espectro Autista.

A pesquisa foi realiza em 7 anais de evento e como resultado principal foram identificas 17 ferramentas assistivas, sendo a maioria contextualizada para o auxílio da alfabetização. A maioria dessas ferramentas são aplicativos, ou seja, voltadas para dispositivos móveis e estão na categoria de jogos educacionais. Com relação a abordagens pedagógicas foram identificadas apenas 5 que foram aplicadas em $59 \%$ das ferramentas.

Um aspecto relevante é a pouca quantidade de ferramentas que são adaptativas, ou seja, se adequam para cada grau do autismo. Entretanto, quanto às diretrizes computacionais adotadas em seu desenvolvimento, $53 \%$ das ferramentas receberam um atenção especial, sendo levado em consideração os aspectos quanto a interface, padrões de projetos e técnicas.

A limitação desse trabalho foi a falta de buscas em fontes internacionais, que poderiam acrescentar outros resultados à pesquisa, agregando maior valor e confiabilidade. Em contra partida, as fontes nacionais trouxeram resultados satisfatórios e suficientes para responder as questões da pesquisa.

Como trabalhos futuros, espera-se ampliar a pesquisa, por intermédio de buscas em mais fontes e evoluir os questionamentos propostos para que seja possível avaliar quais os métodos de validação mais utilizados nas ferramentas e se os conteúdos 
VIII Congresso Brasileiro de Informática na Educação (CBIE 2019)

Anais do XXX Simpósio Brasileiro de Informática na Educação (SBIE 2019)

propostos foram comparados com métodos tradicionais de ensino. Além disso, desenvolver uma ferramenta que agrega os principais aspectos de relevância para apoiar o ensino e a aprendizagem de crianças do TEA.

\section{Referências}

Abreu, F., Almeida, A., Barreiros, E., Saraiva, J., Soares, S., Araújo, A. e Henrique, G. (2012). Métodos, Técnicas e Ferramentas para o Desenvolvimento de Software Educacional: Um Mapeamento Sistemático. Simpósio Brasileiro de Informática na Educação (SBIE), Rio de Janeiro.

APA (2013). American Psychiatric Association. Diagnostic and Statistical Manual of Mental Disorders. 5th ed. Arlington, VA: American Psychiatric Publishing.

Araújo, W. e Almeida, I. (2009). Processo de Desenvolvimento de Software Educativo: Um Estudo Sobre os Modelos. Jornada de Ensino, Pesquisa e Extensão (JEPEX), Recife.

Farias, E., Cunha, M. e Souza, J. (2015). ABC Autismo: Uma Aplicação Mobile para Auxiliar no Processo Alfabetizador de Crianças com Autismo. Workshops do Congresso Brasileiro de Informática na Educação (CBIE), Maceió.

Ferreira, W., Cordeiro, R., Aguiar, Y., Saraiva, J., Tardif, C. e Galy, E. (2018). Panorama das Publicações Nacionais sobre Autismo, Educação e Tecnologia. Simpósio Brasileiro de Informática na Educação (SBIE), Fortaleza, p. 913-922.

Ishihara, M., Tamanaha, A. e Perissinoto, J. (2016). Compreensão de Ambiguidade em Crianças com Transtorno Específico de Linguagem e Fala e Transtorno do Espectro Autista. Revista Codas, v. 28, n. 6, p.753-757.

Kitchenham, B. and Charters, S. (2007). Guidelines for Performing Systematic Literature Reviews in Software Engineering. 2.3. Relatório Técnico Evidence-Based Software Engineering (EBSE), $\quad$ v. 2.3. Disponível em: <http://citeseerx.ist.psu.edu/viewdoc/summary?doi=10.1.1.117.471>. Acesso em: 15 mar. 2019.

Lima, F. (2013). Music Spectrum: Imersão Musical Para Crianças Com Autismo. 2013. 88 f. Dissertação (Mestrado em Informática) - Instituto de Computação da Universidade Federal do Amazonas, Manaus.

Moura, L., Filho, D., Silva, A., Paiva V., Sales, B., Cavalcante, C. e Queiroz, S. F. (2016). TEO: Uma Suíte de Jogos Interativos para Apoio ao Tratamento de Crianças com Autismo. Simpósio Brasileiro de Informática na Educação (SBIE), Uberlândia, p. 627636.

Petersen, K., Feldt, R., Mujtaba, S. e Matisson, M. (2008). Systematic Mapping Studies in Software Engineering. Evaluation and Assessment in Software Engineering, Bari, Italy.

Powell, S. (1996). The Use of Computers in Teaching People with Autism. National Autistic Society Conference, p. 128-132, London.

Putnam, C. e Chong, L. (2008). Software and Technologies Designed for People with Autism: What do Users Want. International Conference on Computers and Accessibility (ACM), New York, p. 3-10.

Silva, M., Moura, I. e Soares, A. (2017). Uso de Tecnologias Computacionais para o Ensino de Crianças com Transtorno do Espectro Autista: Um Mapeamento Sistemático da Literatura. Simpósio Brasileiro de Informática na Educação (SBIE), Recife, p. 173-182.

Sousa, F., Costa, E. e Castro, T. (2012). WorldTour: Software para Suporte no Ensino de Crianças Autistas. Simpósio Brasileiro de Informática na Educação (SBIE), Rio de Janeiro.

Webber, C., Boff, E. e Bono, F. (2009). Ferramenta Especialista para Avaliação de Software Educacional. Simpósio Brasileiro de Informática na Educação (SBIE), Florianópolis. 